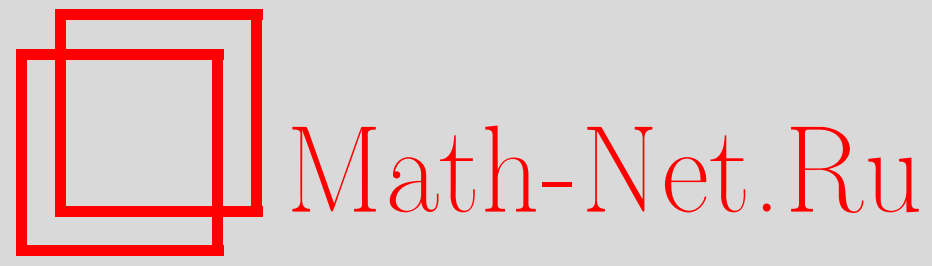

Общероссийский математический портал

Х. Р. Мамедов, О полноте и минимальности половины собственных функций, отвечающих бигармоническому уравнению в полуполосе, Матем. заметки, 1996, том 60, выпуск 3, 460-462

DOI: https://doi.org/10.4213/mzm1851 
Использование Общероссийского математического портала MathNet.Ru подразумевает, что вы прочитали и согласны с пользовательским соглашением

http://www . mathnet.ru/rus/agreement

Параметры загрузки:

IP : 54.172 .240 .79

26 апреля 2023 г., 17:25:04

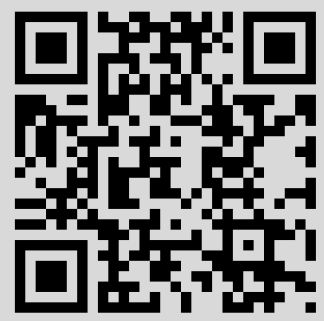




\section{О ПОЛНОТЕ И МИНИМАЛЬНОСТИ ПОЛОВИНЫ СОБСТВЕННЫХ ФУНКЦИЙ, ОТВЕЧАЮЩИХ БИГАРМОНИЧЕСКОМУ УРАВНЕНИЮ В ПОЛУПОЛОСЕ}

\section{Х.P. Мамедов}

Рассмотрим уравнение установившихся колебаний упругой пластинки

$$
\Delta^{2} u(x, y)-\rho \omega^{2} u(x, y)=0
$$

в полуполосе $Q=\{x, y \mid 0 \leqslant x \leqslant 1,0 \leqslant y<\infty\}$ с краевыми условиями защепления на сторонах полуполосы

$$
u(0, y)=u_{x}^{\prime}(0, y)=u(1, y)=u_{x}^{\prime}(1, y)=0
$$

и начальными условиями

$$
u_{y}^{\prime \prime}(x, 0)=\varphi(x), \quad u_{y}^{\prime \prime \prime}(x, 0)=\psi(x),
$$

где $\varphi(x) \in W_{2, U}^{3 / 2}, \psi \in W_{2, U}^{1 / 2}$.

Здесь $\rho$ - плотность пластинки, $\omega$ - частота упругой силы (в статическом случае $\omega=0$ ), а $W_{2, U}^{\theta}$ - замыкание по норме соболевского пространства $W_{2}^{\theta}[0,1]$ $(0 \leqslant \theta \leqslant 4)$ всех функций из $W_{2}^{4}[0,1]$, удовлетворяющих краевым условиям $(2)$.

Целью этой заметки является показать возможность решения этой задачи с помощью разложения по собственным функциям. Различные вопросы, связанные с уравнением (1), изучались во многих работах, близкие по постановке проблемы изучались в работах $[1]-[8]$. 
Разделив переменные $u(x, y)=v(x) e^{i \lambda y}$, перейдем к спектральной задаче

$$
\begin{gathered}
\lambda^{4} v-2 \lambda v^{\prime \prime}+v^{(4)}-\omega^{2} v=0 \\
v(0)=v^{\prime}(0)=v(1)=v^{\prime}(1)=0
\end{gathered}
$$

Для простоты, далее, предположим, что все вещественные собственные значения задачи (4), (5) простые, т.е. $\omega$ не является резонансной частотой. Отметим, что в статическом случае $\omega=0$ вещественные собственные значения отсутствуют вовсе. При $\omega>0$ на вещественной оси находится не более конечного числа собственных значений (см. $[7, \S 9])$. Пусть $\left\{\lambda_{k}^{+}\right\}_{1}^{N}$ - положительные собственные значения, a $v_{k}^{+}(x)$ - собственные функции задачи $(4),(5)$. Обозначим через $M_{+}$конечномерное подпространство, состоящее из незатухающих по $y$ элементарных решений вида $v_{k}^{+}(x) e^{i \lambda_{k}^{+} y}$

Теорема 1. Задача (1)-(3) имеет, причем единственное, решение $u(x, y) \in W^{4}(Q)$, представимое в виде

$$
u(x, y)=u_{0}(x, y)+u^{+}(x, y)
$$

әде $u_{0}(x, y)$ убывает по у әкспоненциально, а $u^{+}(x, y) \in M_{+}$.

ДокАЗАТЕЛЬство. Согласно результатам $\S 8$ работы [1] достаточно доказать существование и единственность убьвающего решения задачи (1)-(3) при $\omega=0$, когда вещественные собственные значения задачи (4), (5) отсутствуют. Далее, повторив прием, примененный в предложении 9.3 работы [7], сведем доказательство к оценке

$$
\|\| u(x, y)\|\| \leqslant C\left(\left\|u_{x}^{(4)}(x, y)\right\|+\left\|u_{y}^{(4)}(x, y)\right\|\right)+C_{1}\|u(x, y)\|,
$$

где ||$|\cdot|||-$ норма в $W_{2}^{4}(Q),\|\cdot\|$ - норма в $L_{2}(Q)$, а оценка предполагается выполненной для функций $u \in W_{2}^{4}(Q)$, удовлетворяющих краевым условиям (2) и нулевым начальным условиям $u_{y}^{\prime \prime}(x, 0)=u_{y}^{\prime \prime \prime}(x, 0)=0$.

Согласно теореме 9.1 работы [7] оценки (6) при $\omega=0$ выполнены в том и только том случае, когда нули вспомогательных задач В. А. Кондратьева, построенных для задачи (1), (3) в угловых точках полуполосы $Q$ не имеют нулей на прямой $\operatorname{Im} \sigma=3$ в комплексной $\sigma$-плоскости (см. $[9, \S 9])$. Угловые точки $Q$ для рассматриваемой задачи симметричны, поэтому для доказательства теоремы остается доказать следующий результат.

Лемма. Нули вспомогательной спектральной задачи В. А. Кондратьева, отвечающей угловой точке $(0,0)$ задачи (1)-(3), не имеют собственных значений на прямой $\operatorname{Im} \sigma=3$.

ДоКАЗАТЕЛЬСтво. Легко убедиться, что вспомогательная задача В. А. Кондратьева для (1)-(3), отвечающая угловой точке $(0,0)$, имеет вид (см. [8])

$$
\begin{gathered}
\left(\sigma^{4}-4 i \sigma^{3}-4 \sigma^{2}\right) \tilde{u}+\left(4+4 i \sigma-2 \sigma^{2}\right) \tilde{u}^{\prime \prime}+\tilde{u}^{(4)}=0 \\
\left\{\begin{array} { l } 
{ \tilde { u } ( 0 ) = 0 , } \\
{ \tilde { u } ^ { \prime } ( 0 ) = 0 , }
\end{array} \quad \left\{\begin{array}{l}
\tilde{u}^{\prime \prime}(\pi / 2)-i \sigma \tilde{u}(\pi / 2)=0 \\
\tilde{u}^{\prime \prime \prime}(\pi / 2)-i \sigma \tilde{u}^{\prime}(\pi / 2)=0
\end{array}\right.\right.
\end{gathered}
$$


Положив $z=i \sigma+1$, можно явно вычислить характеристический определитель указанной задачи, а именно

$$
\begin{aligned}
\Delta(\sigma)= & (z-1)\left[-2 z^{5}-10 z^{4}-20 z^{3}-40 z^{2}-2 z+2\right. \\
& \left.-2\left(z^{5}-z^{4}-6 z^{3}-4 z^{2}+9 z-3\right) \sin ^{2} \frac{z \pi}{2}\right] .
\end{aligned}
$$

Положим $\sigma=b+3 i$ и обозначим через $T(b)$ выражение в квадратных скобках, получающееся после подстановки $z=-2+i b$. Легко убедиться, что

$$
\begin{aligned}
T(b)=T_{1}(b)+i T_{2}(b)= & 10 b^{4}-90+c(b)\left(-11 b^{4}+72 b^{2}-37\right) \\
& +i\left(-2 b^{5}+20 b^{3}+78 b+c(b)\left(b^{5}-42 b^{3}+65 b\right)\right),
\end{aligned}
$$

где $c(b)=-1+\operatorname{ch} b \pi$.

Равенство $T(b)=0$ при $b \in \mathbb{R}$ влечет

$$
\left(10 b^{4}-90\right)\left(b^{4}-42 b+65\right)=-\left(11 b^{4}+72 b^{2}-37\right)\left(-2 b^{4}+20 b^{2}+78\right) .
$$

Но последнее уравнение эквивалентно

$$
3 b^{8}+14 b^{6}+24 b^{4}+274 b^{2}+741=0,
$$

которое вещественных корней не имеет.

Теорема 2. Пусть $\Lambda^{+}$- мнохсество собственных значений задачи (4), (5), лехсащих в открытой верхней полуплоскости и вещественных положстельных. Тогда система производных по М.В. Келдышу иепочек

$$
\hat{v}_{k}^{+}=\left(v_{k}, \lambda_{k}, v_{k}\right), \quad \lambda_{k} \in \Lambda^{+},
$$

отвечающих половине собственных функций, образует полную и минимальную систему в пространстве $W_{2, U}^{3 / 2} \times W_{2, U}^{1 / 2}$.

Доказательство получается применением теоремы 1 , а также теорем 8.6 и 8.7 работы [7].

ЗАМЕчАнИЕ. Учитьвая предложение 9.3 из [7] и теорему 2 с помощью теоремы интерполяции получим полноту и минимальность системы $\left\{v_{k}^{+}\right\}$в пространствах $W_{2, u}^{\theta+1} \times W_{2, u}^{\theta}$ при $1 / 2 \leqslant \theta \leqslant 5 / 2$.

В заключение автор благодарит профессора А. А. Шкаликова за постановку задачи и ценные замечания.

Московский государственный университет им. М. В. Ломоносова

\section{СПИСОК ЦИТИРОВАННОЙ ЛИТЕРАТУРЫ}

1. Ворович И. И. // Труды II Всесоюзного съезда по теоретической и прикладной механике. Вып. 3. М.: Наука, 1966. С. 116-136. 2. Ворович И. И., Ковальчук В.Е. // ПММ. 1967. Т. 31. № 5. С. 861-869. 3. Ковальчук В. Е. // ПММ. 1969. Т. 33. №3. С. 511-518. 4. Устинов Ю. А., Юдович Ю. И. // ПММ. 1973. Т. 73. № 4. С. 706-714. 5. Шкаликов А. А. // Неклассические задачи уравнений математической физики. Новосибирск, 1982. С. 171-174. 6. Шкаликов А. А. // Тр. семинара им. И. Г. Петровского. 1983. Т. 9. С. 190-229. 7. Шкаликов А. А. // Тр. семинара им. И. Г. Петровского. 1989. Т. 41. С. 140-224. 8. Гомилко А. М., Милешко В. А. // Изв. АН СССР. Механика тв. тела. 1986. № 4. C. $48-53$. 